\title{
3D-Morphology Reconstruction of Nanoscale Phase-Separation in Polymer Memory Blends
}

\author{
Vsevolod Khikhlovskyi, Albert J. J. M. van Breemen, Jasper J. Michels, Rene A. J. Janssen, \\ Gerwin H. Gelinck and Martijn Kemerink
}

\section{Linköping University Post Print}

\section{Tweet}

N.B.: When citing this work, cite the original article.

Original Publication:

Vsevolod Khikhlovskyi, Albert J. J. M. van Breemen, Jasper J. Michels, Rene A. J. Janssen, Gerwin H. Gelinck and Martijn Kemerink, 3D-Morphology Reconstruction of Nanoscale Phase-Separation in Polymer Memory Blends, 2015, Journal of Polymer Science Part B: Polymer Physics, (53), 17, 1231-1237.

http://dx.doi.org/10.1002/polb.23769

Copyright: Wiley: 12 months http://eu.wiley.com/WileyCDA/

Postprint available at: Linköping University Electronic Press http://urn.kb.se/resolve?urn=urn:nbn:se:liu:diva-120640 


\section{D-morphology reconstruction of nanoscale phase-separation in polymer memory blends.}

\section{Vsevolod Khikhlovskyi, Albert J.J.M. van Breemen, Jasper J. Michels, René A.J. Janssen, Gerwin H. Gelinck and Martijn Kemerink}

V. Khikhlovskyi, Prof. Dr. M. Kemerink, Prof. Dr. G.H. Gelinck, Prof. Dr. R.A.J. Janssen, Department of Applied Physics, Eindhoven University of Technology,

P. O. Box 513, $5600 \mathrm{MB}$ Eindhoven, The Netherlands

Prof. Dr. M. Kemerink,

Department of Physics, Chemistry and Biology (IFM),

Linköping University,

P. O. Box SE-581 83 Linköping, Sweden

V. Khikhlovskyi, Dr. A.J.J.M. van Breemen, Prof. Dr. G.H. Gelinck, Holst Centre, TNO-The Dutch Organization for Applied Scientific Research, High Tech Campus 31, 5656 AE Eindhoven, The Netherlands

Dr. Jasper Michels, Max Planck Institute for Polymer Research (MPI),

Ackermannweg 10 - D-55128 Mainz,

P.O. Box 3148 - D-55021 Mainz, Germany

Correspondence to: Martijn Kemerink (E-mail: martijn.kemerink@liu.se), Gerwin H. Gelinck (E-mail: gerwin.gelinck@tno.nl)

((Additional Supporting Information may be found in the online version of this article.))

\section{ABSTRACT}

In many organic electronic devices functionality is achieved by blending two or more materials, typically polymers or molecules, with distinctly different optical or electrical properties in a single film. The local scale morphology of such blends is vital for the device performance. Here, a simple approach to study the full 3D morphology of phase-separated blends, taking advantage of the possibility to selectively dissolve the different components is introduced. This method is applied in combination with AFM to investigate a blend of a semiconducting and ferroelectric polymer typically used as active layer in organic ferroelectric resistive switches. It is found that the blend consists of a ferroelectric matrix with three types of embedded semiconductor domains and a thin wetting layer at the bottom electrode. Statistical analysis of the obtained images excludes the presence of a fourth type of domains. The criteria for the applicability of the presented technique are discussed.

KEYWORDS: selective dissolution, phase separation, AFM, thin film, organic memory 


\section{INTRODUCTION}

Soluble organic semiconductors are receiving increasing attention as they enable emerging applications such as flexible and low-cost organic photovoltaics (OPV) ${ }^{1}$, light emitting diodes (OLED) ${ }^{2}$, field effect transistors (OFET) ${ }^{3}$, and memories. ${ }^{4-8}$ For all these devices, performance can be optimized by blending two components to obtain a (controlled) phase separated morphology. For OPV, the use of separate donor and acceptor moieties is imperative to achieve efficient charge generation. ${ }^{9}$ Likewise, data storage in twoterminal organic ferroelectric resistive memories is intimately related to phase separation between the blend components ${ }^{10}$. 3D morphology control in polymer resistive memories has recently been shown to result in enhanced functional device performance. ${ }^{11,12}$ High-performance uni- and ambipolar OFETs can be made from phase separated polymer blends. ${ }^{13}$ In such blend systems the small molecule component and the polymer binder are responsible for high charge carrier mobility and formation of smooth, uniform films respectively.

Accurate characterization of the blend morphology is challenging due to the relatively high chemical and mechanical similarity of the components and the small length scales involved. ${ }^{14,15}$ Although atomic force microscopy (AFM), transmission/scanning electron microscopy (TEM/SEM), and scanning near-field optical microscopy (SNOM) provide sufficient resolution it is quite difficult for these techniques to recognize different components of the blend. In addition, TEM/SEM and SNOM require specific sample preparation and advanced practical knowledge of the setups. It has to be noticed that in most cases direct measurements by AFM provides information only about the topography of the top surface of the blend. Alternatively, scanning transmission $\mathrm{X}$-ray spectroscopy (STXM) can be used to study the blend morphology, but it requires synchrotron access. ${ }^{10}$ Therefore a strong demand exists for a simple and an easily accessible method that can provide detailed information about the 3D-structure of organic blend morphologies at the local scale.

A system in which there is a clear need for enhanced insight in the 3D morphology is the organic ferroelectric resistive memory switch. ${ }^{4-8}$ The resistivity of such memories can be switched between two distinct levels that retain their states when the power is shut off. Another important feature of the memory is the possibility of non-destructive read out, making the organic ferroelectric resistive switch an extremely promising candidate for low-cost non-volatile reprogrammable memory applications. ${ }^{16}$ Its active layer consists of a phase-separated blend of a ferroelectric and semiconducting polymer processed from a common solution. During casting and solvent evaporation the final blend morphology forms via spinodal decomposition. ${ }^{17,18}$ The final dry film consists of semiconducting domains embedded in a polycrystalline ferroelectric matrix. ${ }^{10}$ Transport of charge carriers occurs in the semiconducting phase. The underlying switching mechanism is based on a modulation of the injection barrier by the stray field of the ferroelectric polarization charges in the vicinity of the ferroelectric/semiconductor interface. ${ }^{19}$, 20 It has been shown that the operation of ferroelectric resistive switches strongly depends on the 3D-morphology of the phase-separated blend. ${ }^{10,17}$ Hence detailed information about the blend structure is not only crucial for complete understanding, but also for further optimization and miniaturization.

Here we present a combined technique of selective dissolution and statistical analysis - a simple and powerful tool for studying the 3D structure of the blend at submicron length scales. It takes advantage of selective solubility of different components of the blend. Hence different components of the blend are selectively dissolved and the remaining structures together with the as-processed blend are studied by AFM. Combination of all 
measured topography images gives the possibility to reconstruct the 3D-structure of the blend. The technique should be applicable to any demixed blend system based on components with sufficiently mutual repulsion to allow for selective dissolution and a morphology that is 'sufficiently coarse'; more specific criteria are discussed at the end.

\section{EXPERIMENTAL}

Poly[(9,9-di-n-octylfluorenyl-2,7-diyl)-alt (benzo[2,1,3]thiadiazol-4,8-diyl)] (F8BT) $\left(M_{n}=\right.$ $4.9 \mathrm{kDa}, \mathrm{D}=1.8$ ) was synthesized according to a modified Suzuki polymerization. ${ }^{21,22}$ The ferroelectric polymer, Poly(vinylidene fluorideco-trifluoro ethylene) (P(VDF-TrFE)) $\left(M_{n}=220\right.$ $\mathrm{kDa}, 2.3<\mathrm{D}<2.8$ with a 77/23 VDF/TrFe ratio) was supplied by Solvay Specialty Polymers. Blend solutions were prepared by co-dissolving P(VDF-TrFE) and F8BT (9:1 w/w) in a mixture of cyclohexanone and tetrahydrofurane 75:25 (v/v) at $50{ }^{\circ} \mathrm{C}$ overnight. Cleaned glass substrates with litho patterned gold were used. Blend films were then spin coated in a nitrogenfilled glove box. The average thickness of the film was measured with a DEKTAK profilometer and found to be $\sim 200 \mathrm{~nm}$. Subsequently, the samples were annealed at $135^{\circ} \mathrm{C}$ for 1 hour.

For the dissolution technique dimethylsulfoxide (DMSO) and toluene were used to selectively dissolve the ferroelectric (P(VDF-TrFE)) and the semiconductor (F8BT) parts of the blend respectively.

Surface topography of the dried blend films was examined by atomic force microscopy (AFM, Veeco MultiMode NS-IIIA). Topography is measured using a sharp (apex radius $<10 \mathrm{~nm}$ ) AFM tip in tapping-mode. The AFM measurements were performed using Al coated Si tips (Nanosensors, spring constant $\mathrm{k} \sim 12.5$ $\mathrm{N} / \mathrm{m}$ ). The C-AFM current was detected using a high sensitivity current amplifier (TUNA module, $10^{11} \mathrm{~V} / \mathrm{A}$, Veeco) together with $\mathrm{Pt} / \mathrm{Ir}$ coated $\mathrm{Si}$ tips (Nanosensors, spring constant $\mathrm{k} \approx 5 \mathrm{~N} / \mathrm{m}$ ).
Statistical analysis of the selective dissolution data was performed using commercial image analysis software Image-Pro Premier. Diameter and height of each domain, as well as of the (partial) wetting layer at the bottom surface, were identified by the software based on pixel intensity values.

\section{RESULTS AND DISCUSSION}

The system studied in this work is a continuous film of a 1:9 wt/wt blend of the polymeric semiconductor poly(9,9-di-n-octylfluorene-altbenzothiadiazole) (F8BT), and the ferroelectric polymer poly(vinylidene fluoride-co-trifluoro ethylene) (P(VDF-TrFE)), with $77 \mathrm{~mol} \%$ VDF. The film was spincoated from $50 \mathrm{mg} \mathrm{mL}^{-1}$ a cyclohexanone $(\mathrm{CH})$ and tetrahydrofuran (THF) mixture $(75: 25 \mathrm{v} / \mathrm{v})$ on top of glass substrates with gold electrodes (3000 rpm, $60 \mathrm{sec}$ ) and annealed at $135{ }^{\circ} \mathrm{C}$ for 1 hour to enhance the crystallinity of the ferroelectric phase. Using surface profilometry we determined the average layer thickness of the P(VDF-TrFE):F8BT blend to be $\sim 200 \mathrm{~nm}$ and the rms (root mean square) roughness to be below $20 \mathrm{~nm}$. All technical details of the selective dissolution procedure can be found in the experimental section and in Ref. 11. As has been proposed for this particular system ${ }^{11}$ as well as for such blends with other polymeric semiconductors, like $\mathrm{P}_{3} \mathrm{HT}^{4,5}$ and $\mathrm{PFO}^{23}$, the ternary blend of solvent/semiconductor/P(VDF-TrFE) demixes during solvent evaporation to give (for a 1:9 w/w F8BT:P(VDF-TrFE) ratio) a dispersed phase of disk-like domains consisting of virtually pure semiconductor in a matrix of $\mathrm{P}(\mathrm{VDF}-\mathrm{TrFE}){ }^{10}$ Despite the fact that this is generally true for all polymeric semiconductors used so far in combination with P(VDF-TrFE), subtle differences remain.

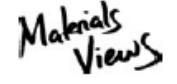

WWW.MATERIALSVIEWS.COM 


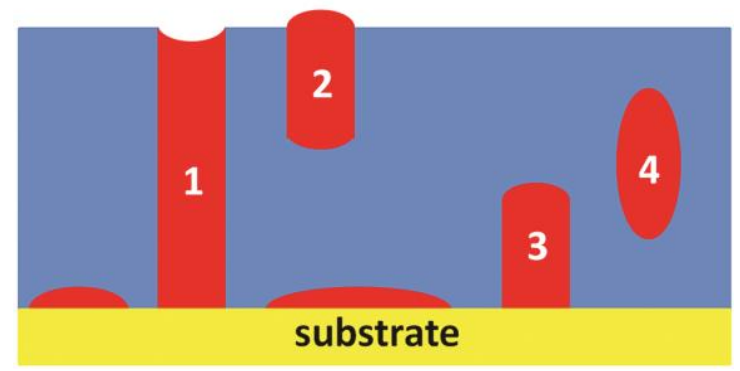

FIGURE 1 Schematic cross-sectional representation of the $\mathrm{P}(\mathrm{VDF}-\mathrm{TrFE})$ :F8BT blend film on gold; F8BT and P(VDF-TrFE) phases are indicated in red and blue, respectively.

In case of $\mathrm{P}(\mathrm{VDF}-\mathrm{TrFE})$ : $\mathrm{FBBT}$ processed from cyclohexanone/THF on gold, the blend films typically exhibit four types of semiconducting domains ${ }^{11,15}$ (see Figure 1); i) electrically functional "Type 1" domains that locally bridge the total thickness of the film (see Figure S1), ii) electrically non-functional "Type 2" domains locally protruding from the top region of the ferroelectric matrix but not connecting to the Au substrate, iii) electrically non-functional "Type 3" domains, that reside on the substrate but are buried by the matrix phase, iv) fully embedded "Type 4" domains, and v) very thin $(<30 \mathrm{~nm})$ regions on the substrate comprising F8BT, reminiscent of a "partial wetting layer". As the wetting layer can potentially form a semi-continuous layer it is treated separately and not as another domain type. Since the substrate was constantly moved during the dissolution process and flushed with solution afterwards, Type 2 and 4 domains were prevented to adhere to the substrate and being wrongly interpreted as Type 3 domains. In passing we note that the observed concave top of Type 1 domains has a beneficial effect on the device performance. On basis of numerical simulations it was found that domains with a concave top yield enhanced switching compared those with convex or flat tops due to the closer proximity of the injection point to the ferroelectric polarization charges. ${ }^{24}$ In the AFM topography images of the P(VDF-TrFE):F8BT blend in Figure 2, the first three domain types can all be identified. The figure shows height scans of the as-processed blend film (Figure 2a), taken after selective removal of the ferroelectric matrix (Figure 2b), and upon selective removal of the F8BT material that is accessible from the top surface of the film. The exact procedure followed to selectively dissolve the phases is mentioned in the Experimental Section. The elegance of selective phase dissolution is that the resulting topology images provide complementary pieces of information that together allow reconstruction of the full 3D morphology of the phase separated blend film.
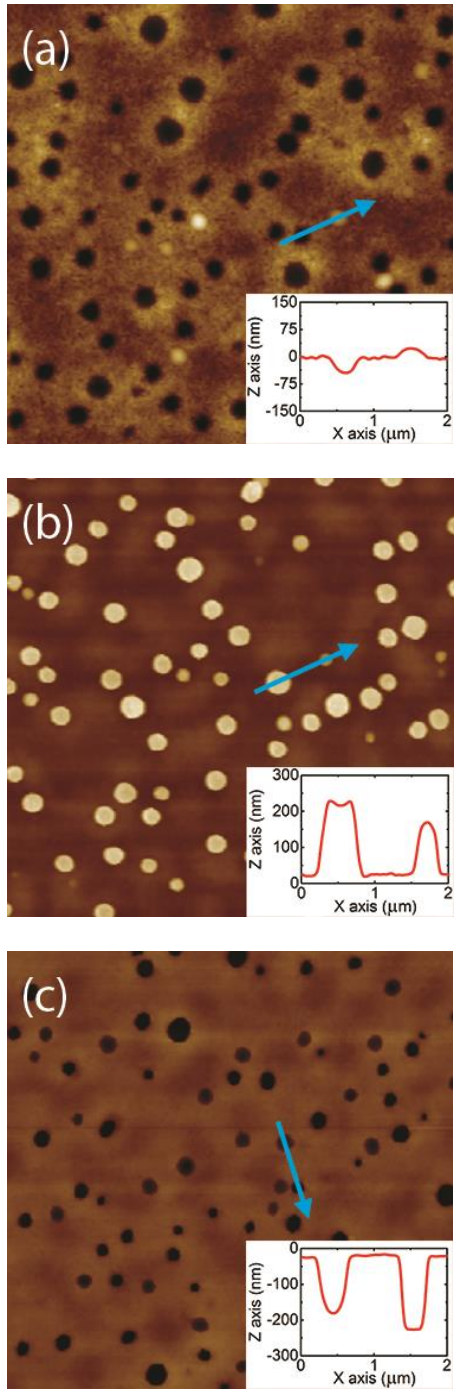

FIGURE 2 Selective dissolution method applied on a P(VDF-TrFE):F8BT (9:1 w/w) blend deposited on a Au substrate. AFM topography 
images $\left(10 \times 10 \mu \mathrm{m}^{2}\right)$ of: (a) as processed blend. (b) the remaining structure of the blend after selectively dissolving the ferroelectric phase. (c) the remaining structure of the blend after selectively dissolving the semiconductor (F8BT) phase. Insets indicate line sections over the blend shown with blue arrows. The total vertical scale is $300 \mathrm{~nm}$ in all panels.

From Figure 2a (blend film), together with conductive force microscopy performed previously $^{20}$ (Figure S1), we deduce that the electrically active Type 1 domains are situated within the concave ('dark') circular areas, i.e. below the average height of the matrix phase. The circular convex ('light') shapes in Figure 2a correspond to Type 2 domains, which protrude from the film but do not contact the substrate. The concave top surface of Type 1 domains that is visible in the cross-sectional scan (inset figure 2a) seems confirmed by Figure $2 b$ (matrix removed). In contrast, the lower features in Figure $2 \mathrm{~b}$ are convex and assigned to be Type 3 domains, i.e. initially covered by the P(VDFTrFE) and hence not visible in Figure 2a. It is important that the shape of the side part of the domains is affected by the convolution with the AFM tip and reflects the opening angle of the probe. However, the geometry of the tip together with extremely slow scanning speed allows easy detection of all types of the domains and cavities associated with them. We note that removal of the matrix phase leads to the removal of the Type 2 and Type 4 domains in Figure $2 b$ and therefore does not allow their detection.

The occurrence of Type 1 and Type 2 domains is further supported by Figure 2c (F8BT removed). Of the circular holes resulting after selective dissolution only the deeper ones have a flat bottom surface, strongly indicating the hole to persist all the way down to the substrate and to have been occupied by a Type 1 F8BT domain. In contrast, holes with relatively small diameters do not extend towards the substrate (see inset Figure 2c), and would therefore have been occupied by a Type 2 domain. It is noted that the selective dissolution procedure does not allow for direct identification of Type 4 domains, as they would be undetectable in the pristine blend film, screened against selective dissolution, and removed unselectively upon matrix dissolution.

The images in Figure 2 show that most of the semiconducting material resides in the lower region of the blend film. This finding, together with the appearance of low contrast features in Figure $2 b$ that we interpret as a "partial wetting layer" of F8BT, indicates a favorable interaction between F8BT and gold. ${ }^{25}$ The non-isotropic distribution of the semiconducting material along the z-axis suggests the aforementioned spinodal decomposition ${ }^{26}$ to be surfacedirected. It is thought that an initially lamellar morphology resulting from pronounced stratification breaks up into a droplet-like morphology during later stages. To what extent demixing is surface-directed depends on i) the difference in interaction strength of the individual blend components with the air- and substrate interfaces and ii) the ratio of the diffusive and evaporative time scales. Once break-up of the lamellae has occurred, interfacial forces would prevent the reformation of elongated fluid structures. For this reason it is not surprising that the Type 1 domains are both higher and wider than the Type 3 (or Type 2) domains.

Concave features in the Type 1 domains may be explained in terms of the difference in solvent compatibility of the polymeric blend components. ${ }^{27}$ They originate in the final stages of drying of the blend, during which the more diluted phase collapses around, or in between structures representing the already vitrified concentrated phase. An alternative explanation for the concave nature of the Type 1 domains may be resumed wetting of the gold substrate by F8BT during annealing. The elevated temperature can potentially reintroduce some mobility to the blend components, possibly allowing for F8BT structures to slowly spread 
onto the gold substrate and locally extend under the P(VDF-TrFE) matrix. This might also explain the fact that the matrix is consistently elevated at the edge of the Type 1 domains.

It is important to point out that the presence of the Type 1 domains is crucial for the device performance. In contrast, the electrically nonaddressable Type 2, Type 3, Type 4 domains and the wetting layer represent a significant waste of the semiconductor material in the blend.

The presence of the Type 1 and Type 2 domains was independently confirmed by a combination of Scanning Probe Microscopy techniques, including conductive-AFM, on the same blend ${ }^{20}$. In Ref. 20 and in the $\mathrm{SI}$ it is shown that only Type 1 domains are responsible for the current in the device whereas Type 2 domains carry no measurable current.

Having identified and confirmed the existence of various types of domains, we proceed with a quantitative assessment of the amount of material in each of the different phases. For the memory diode this is an important measure for the effective active area of the device and for the potential for improvement (by avoiding non-Type 1 domains). In order to estimate the volume fraction of electrically functional F8BT material in the blend films (i.e. represented by the Type 1 domains), we subjected the AFM topography data corresponding to Figures $2 b$ and $2 \mathrm{c}$ to statistical analysis in terms of domain diameter and height. A statistically relevant sample size was obtained by considering a field of view of $20 \times 20 \mu \mathrm{m}^{2}$, rather than $10 \times 10 \mu \mathrm{m}^{2}$ (see Figure S2). Statistical data was obtained by quantitative analysis of the digital AFM images using commercial image processing software Image-Pro Premier. Figures 3 and 4 show the resulting distribution functions - probability density function (PDF) and cumulative distribution function (CDF) for domain height and diameter (or hole depth and diameter), as well as a graph wherein the height (depth) of individual domains (holes) has been plotted against diameter. It has to be noted that the domain height in Figure 3 as well the hole depth in Figure 4 correspond to the average values obtained from the AFM topography data. Both figures represent the same types of the domains mentioned above, and are in agreement with the film thickness.
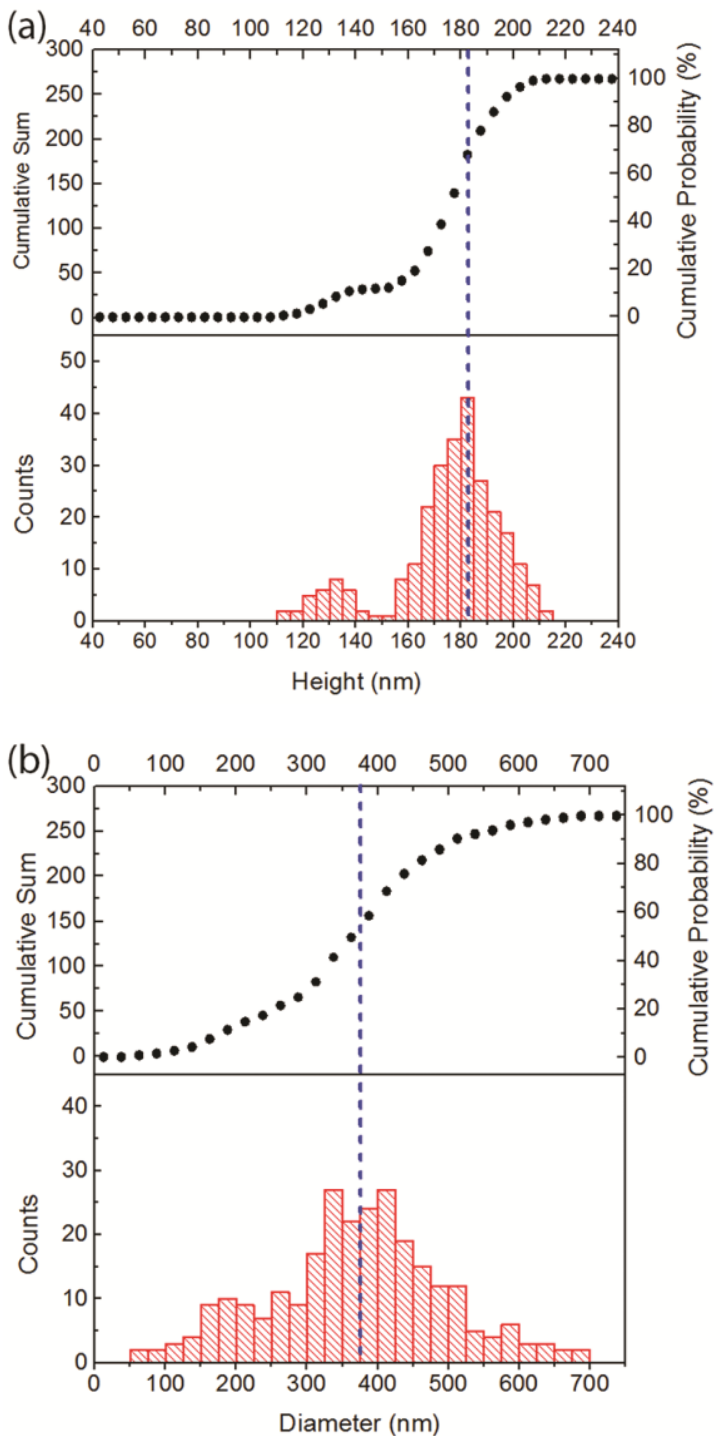


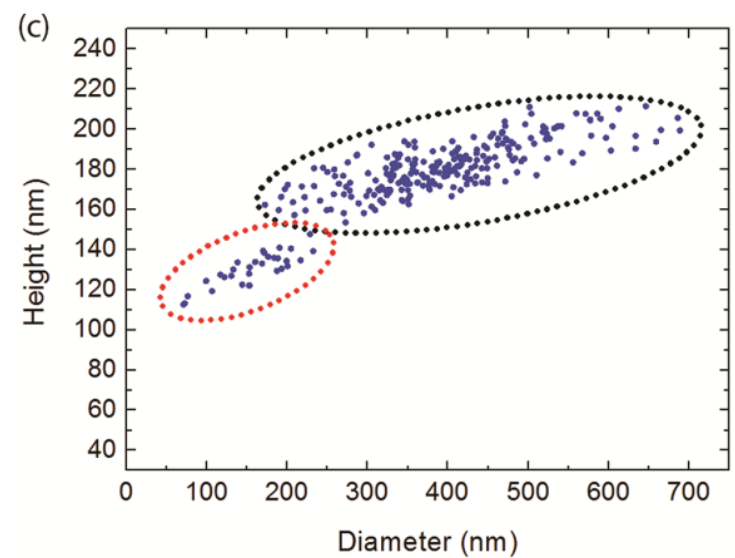

FIGURE 3 Statistical analysis of the AFM images $\left(20 \times 20 \mu^{2}\right)$ (Figure S2b) taken on selectively dissolved P(VDF-TrFE) phase of a P(VDFTrFE):F8BT (9:1 w/w) blend on Au electrode shown in Figure 2b. (a). Domain height distribution (histogram, cumulative probability and cumulative sum). (b). Domain diameter distribution (histogram, cumulative probability and cumulative sum). (c). Plot of domain diameter vs. domain height. Black and red dashed ellipses represent two distinct clusters of points which correspond to the Type 1 and Type 3 domains respectively.

The bell-shaped distribution functions (Figure $3 a, 3 b)$ clearly demonstrate the presence of dominant length scales in the analyzed morphologies, which is indicative for spinodal demixing. Interestingly, the distribution functions are bimodal: two characteristic length scales (domain sizes) are observed. We recall that for "classical" thermally quenched systems spinodal decomposition is characterized by only a single dominant length scale but numerical simulations exclusively based on diffusive dynamics have recently shown the emergence of a second dominant length scale in spinodally demixed polymer/polymer/solvent and polymer/solvent systems to result from mass redistribution as a consequence of solvent evaporation. ${ }^{28}$

The positive tilt with respect to the horizontal axis of the ellipses marked in Figures $3 c$ and $4 c$ illustrate the remark made above that the highest domains are also expected to have the largest diameter, as interfacial forces limit the aspect ratio of structures in the demixed fluid.

An important finding in Figure $3 \mathrm{c}$ and Figure $4 \mathrm{c}$ is the fact that the data points form two distinct clusters. Detailed statistical analysis of the data and comparison with the AFM topography images suggests that the majority of each type of the domains $(1,2$ or 3 , see Fig. 1$)$ reside in a particular cluster. This gives the possibility to estimate the total volume occupied by the specific type of the domains. For simplicity, for the volume calculation every domain was considered to be cylindrically-shaped with flat top and bottom parts.
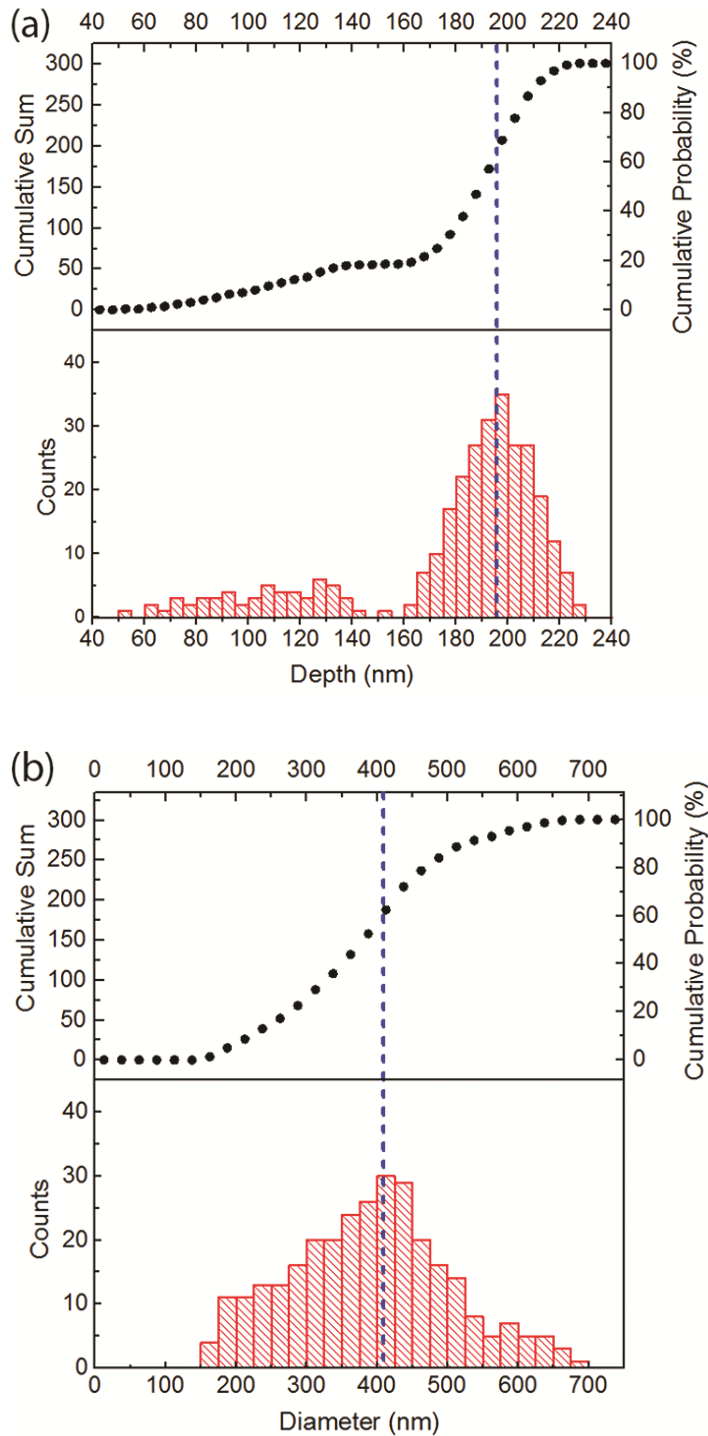


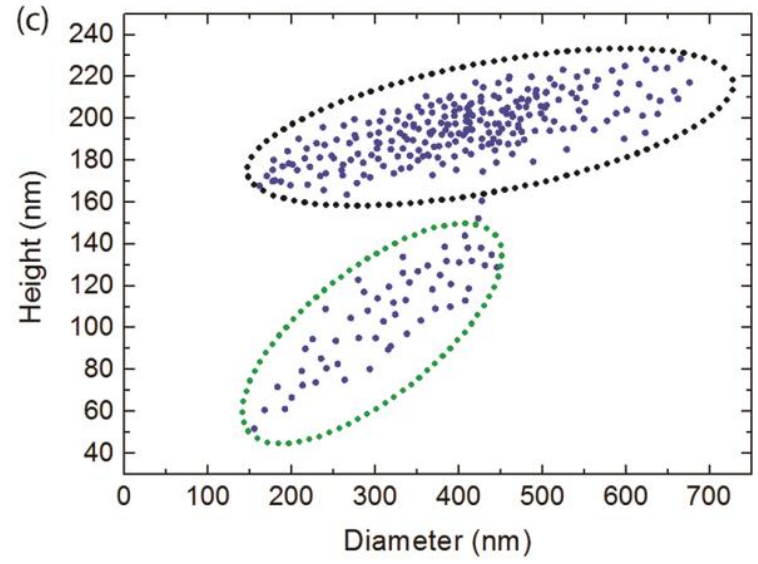

FIGURE 4 Statistical analysis of the AFM images $\left(20 \times 20 \mu^{2}\right.$ ) (Figure S2c) taken on selectively dissolved F8BT phase of a P(VDF-TrFE):F8BT (9:1 $\mathrm{w} / \mathrm{w}$ ) blend on Au electrode shown in Figure 2c. (a). Hole depth distribution (histogram, cumulative probability and cumulative sum). (b). Hole diameter distribution (histogram, cumulative probability and cumulative sum). (c). Scatter plot of hole diameter vs. hole depth. Black and green dashed ellipses represent two distinct clusters of points which correspond to the Type 1 and Type 2 domains respectively.

The volume fraction of each type of the domains was calculated based on the initial volume of the semiconductor polymer in the initial solution and the average thickness of the blend. The Type 1, Type 2, Type 3 domains and the wetting layer yield around $67 \pm 0.5 \%, 2 \pm$ $0.5 \%, 8 \pm 0.5 \%$ and $23 \pm 0.5 \%$ of the total volume of the semiconductor respectively. The fact that these add up to $\sim 100 \%$ excludes the existence of Type 4 domains. Thus, by combining topographical images with a full statistical analysis, the selective dissolution method provides detailed information about the 3D-structure of the blend at the local scale. It has to be noted that the reconstructed blend structure is in a good agreement with previously studied similar blend - P(VDFTrFE):P3HT. ${ }^{10,17}$ It has to be mentioned that the presence of the vertical, columnar-like domains, i.e. Type 1-like, in that blend was also found by SEM, SNOM ${ }^{19}$ and STXM ${ }^{10}$ techniques.
We shall now briefly address the requirements that should be met by the phase separated blend to enable the application of the presented selective dissolution technique. First, the need for selective solvents does not imply the necessity of pure phase domains even though the presence of mixed phase domains will make it harder to find orthogonal solvents. Second, it is clear that the feature sizes in the present system are far from the ultimate limit for the technique. From an AFM perspective minimum feature sizes could in principle be in the nanometer range. However, tip convolution effects associated with typical tip radii around $10 \mathrm{~nm}$ and tip cone angles around $30^{\circ}$ will put an upper limit to the aspect ratio of 'workable' features of around 2, i.e. $50 \mathrm{~nm}$ wide for a 100 $\mathrm{nm}$ high feature. High-aspect ratio tips can push this limit considerably further. From the morphology perspective the major concern is that the dissolution of one phase has to leave a 'representative' topography of the other phase. Hence, remaining features should also be morphologically stable, i.e. should not collapse. This excludes finely intermixed phases of soft materials, as e.g. found in many optimized organic photovoltaic bulk heterojunctions. Finally, the phase separation should lead to mostly bicontinuous phases, i.e. no, or only a small fraction of each phase should be completely surrounded by the other phase, c.f. domain Type 4 in Figure 1 that could be excluded for the present system but would be washed away by dissolving the surrounding P(VDF-TrFE).

\section{CONCLUSIONS}

In summary, we have introduced a selective dissolution approach for studying the 3Dmorphology of phase-separated blends at the local scale. The method takes advantage of the selective solubility of different components of the blend. The two component blend of a semiconductor (F8BT) and a ferroelectric ( $P($ VDF-TrFE)) polymer was investigated in this work. This system is a representative example 
of an active layer in an organic ferroelectric resistive switch. It was found that three distinct types of nearly pure semiconductor domains are present in the blend. A possible fourth type could be ruled out on basis of a statistical analysis. Thus, the selective dissolution approach is a simple and powerful technique that provides information about the 3Dstructure of phase separated blends at the local scale. The method is potentially applicable to a wider range of phase separated blends meeting criteria that are discussed above; roughly spoken, features should be predominantly vertical and have an aspect ratio below $\sim 2$, i.e. $50 \mathrm{~nm}$ wide for a height of $100 \mathrm{~nm}$.

\section{ACKNOWLEDGEMENTS}

The research leading to these results has received funding from the European Community's Seventh Framework Programme (FP7/2007-2013) under grant agreement $n^{\circ}$ 248092 of the MOMA project.

\section{REFERENCES AND NOTES}

1. C. J. Brabec, M. Heeney, I. McCulloch and J. Nelson, Chem. Soc. Rev. 2011, 40, 1185-1199.

2. A. C. Morteani, A. S. Dhoot, J. S. Kim, C. Silva, N. C. Greenham, C. Murphy, E. Moons, S. Ciná, J. H. Burroughes, R. H. Friend, Adv. Mater. 2003, $15,1708-1712$.

3. A. C. Arias, F. Endicott and R. A. Street, Adv. Mater. 2006, 18, 21, 2900-2904.

4. K. Asadi, D. M. de Leeuw, B. de Boer, and P. W. M. Blom, Nature Mater. 2008, 7, 547-550.

5. K. Asadi, T. G. de Boer, P. W. M. Blom, D. M. de Leeuw, Adv. Funct. Mater. 2009, 19, 3173-3178.
6. J. Hu, J. Zhang, Z. Fu, J. Weng, W. Chen, S. Ding, Y. Jiang, and G. Zhu, ACS Appl. Mater. Interfaces 2015, 7, 6325-6330.

7. M. A. Khan , U. S. Bhansali , D. Cha , and H. N. Alshareef, Adv. Funct. Mater. 2013, 23, 21452152.

8. T. Braz, Q. Ferreira, A. L. Mendonca, A. M. Ferraria, A. M. B. do Rego, and J. Morgado, J. Phys. Chem. C 2015, 119, 1391-1399.

9. R. A. J. Janssen and J. Nelson, Adv. Mater. 2013, 25, 1847-1858.

10. C. R. McNeill, K. Asadi, B. Watts, P. W. M. Blom and D. M. de Leeuw, Small 2010, 6, 4, 508512.

11. A. van Breemen, T. Zaba, V. Khikhlovskyi, J. Michels, R. Janssen, M. Kemerink, and G. Gelinck, Adv. Funct. Mater. 2015, 25, 278-286.

12. S. H. Sung and B. W. Boudouris, ACS Macro Lett. 2015, 4, 293-297.

13. X. Yang, Semiconducting Polymer Composites: Principles, Morphologies, Properties and Applications, WILEY-VCH, 2012; Chapter 7, pp 191-251.

14. X. Yang, J. Loos, S. C. Veenstra, W. J. H. Verhees, M. M. Wienk, J. M. Kroon, M. A. J. Michels, and R. A. J. Janssen, Nano Lett. 2005, 5, 4, 579-583.

15. S. S. van Bavel, E. Sourty, G. de With and J. Loos, Nano Lett. 2009, 9, 2, 507-513.

16. A. J. J. M. van Breemen, J.-L. van der Steen, G. van Heck, R. Wang, V. Khikhlovskyi, M. Kemerink, and G.H. Gelinck, Appl. Phys. Express, 2014, 7, 031602.

17. K. Asadi, H. J. Wondergem, R. S. Moghaddam, C. R. McNeill, N. Stingelin, B. Noheda, P. W. M. Blom, and D. M. de Leeuw, Adv. Funct. Mater. 2011, 21, 1887-1894. 
18. J. J. Michels, A. J. J. M. van Breemen, K. Usman, G. H. Gelinck, J. Polym. Sci. Part B: Polym. Phys. 2011, 49, 1255-1262.

19. M. Kemerink, K. Asadi, P. W. M. Blom, D. M. de Leeuw, Org. Electron. 2012, 13, 147-152.

20. V. Khikhlovskyi, R. Wang A. J. J. M. van Breemen, G. H. Gelinck, R. A. J. Janssen, and M. Kemerink, J. Phys. Chem. C, 2014, 118, 33053312.

21. T. van Woudenbergh, J. Wildeman, P. W. M. Blom, J.J. A. M. Bastiaansen, B. M. W. Langeveld-Voss, Adv. Funct. Mater. 2004, 14, 677-683.

22. N. Miyaura, A. Suzuki, Chem. Rev. 1995, 96, 2457-2483.

23. M. Li, N. Stingelin, J. J. Michels, M.-J. Spijkman, K. Asadi, R. Beerends, F. Biscarini, P.
W. M. Blom, and D. M. de Leeuw, Adv. Funct. Mater. 2012, 22, 2750-2757.

24. R. Wang, V. Khikhlovskyi, M. Kemerink, unpublished.

25. A. Budkowski, A. Bernasik, P. Cyganik, J. Rysz, R. Brenn, e-Polymers 2002, 6, 1-21.

26. C. C. Lin, H. S. Jeon, N. P. Balsara, B. Hammouda, J. Chem. Phys. 1995, 103, 5, 19571971.

27. S. Walheim, M. Bölltau, J. Mlynek, G. Krausch, and U. Steiner, Macromolecules 1997, 30, 4995-5003.

28. C. Schaefer, P. van der Schoot, and J. J. Michels, Phys. Rev. E 2015, 91, 022602. 